\title{
ANTAGONISMO DI TRICHODERMA SPP AI FITOPATOGENI CHE CAUSANO MARCIUME DELLA RADICE DI MANIOCA IN ALAGOAS
}

\section{RECENSIONE ARTICOLO}

SILVA, Izael Oliveira ${ }^{1}$

AMORIM, Edna Peixoto da Rocha ${ }^{2}$

JUNIOR, Nelson Augusto Nascimento ${ }^{3}$

PEIXINHO, Georgia Souza ${ }^{4}$

CARNAÚBA, Juliana Paiva ${ }^{5}$

NETO, Vicente Ferreira de Araujo ${ }^{6}$

SILVA, Izael Oliveira. Et al. Antagonismus von Trichoderma spp. ai fitopatogeni che causano marciume della radice di manioca in Alagoas. Revista Científica Multidisciplinar Núcleo do Conhecimento. Anno 05, Ed. 06, Vol. 12, pp. 149-177.

${ }^{1}$ Dottorato in Protezione Delle piante, Master in Master per la Produzione di Piante, Bachelor e Bachelor in Scienze Biologiche.

${ }^{2}$ Dottorato di ricerca in Agronomia (Agricoltura). Laurea magistrale in Fitopatologia. Specializzazione in Didattica per l'istruzione superiore. Laurea in Agronomia.

${ }^{3}$ Dottorato in Protezione delle piante. Laurea magistrale in Agronomia (Irrigazione e Drenaggio). Laurea in Agronomia

${ }^{4}$ Laurea magistrale in Orticoltura. Laurea in Agronomia.

${ }^{5}$ Dottorato di ricerca in Fitopatologia. Laurea magistrale in Agronomia (Produzione vegetale e protezione delle piante). Miglioramento in Miglioramento delle Scienze Biologiche. Laurea in Agronomia.

${ }^{6}$ Laurea in Agronomia. 
giugno 2020. ISSN: 2448-0959, collegamento di acesso: https://www.nucleodoconhecimento.com.br/agronomia-it/antagonismo-di-trichoderma

\section{RIEPILOGO}

Molti sono fitopatogeni che limitano la produzione di manioca (Manihot esculata Crants) rotei delle radici a cui sposano la più grande perdita nel raccolto raggiungendo il 70\%. Questa cultura è di grande interesse per la regione nord-orientale del Brasile, fornendo grandi perdite. Le difficoltà di gestione hanno diretto la ricerca di alternative, tra le quali i biocontrollori hanno mostrato risultati significativi. Questa revisione della letteratura mirava a raccogliere informazioni relative al marciume della radice di manioca cv Rosinha, i principali fitopatogeni e attività antagoniste del trichoderma nel controllo delle malattie nello stato di Alagoas. Un'indagine bibliografica è stata condotta nelle banche dati Scielo, Google Acad-mico, portale Capes (tesi e tesi), diari e libri. Per ottenere informazioni su: marciume radicale della cassavia tavolo, Trichoderma spp., come agente di biocontrollo dei fitopatogeni.

Parole chiave: radice tuberosa, controllo biologico, fitopatologia.

\section{INTRODUZIONE}

Grazie al suo valore nutrizionale, la Esculenta Manihot Crantz si distingue in Brasile e nel mondo con un significativo contributo economico e sociale grazie alla sua versatilità, in quanto viene utilizzato sia nel cibo animale che umano e ampiamente utilizzato nell'industria. La coltura esercita un sostegno significativo alla popolazione con scarso potere d'acquisto, perché oltre ad essere una fonte nutriente è anche un generatore di reddito nell'agricoltura familiare (NOTARO et al. 2013).

Nel mondo, il consumo di manioca e dei suoi derivati è al sesto posto nella classifica tra soia, grano, riso, mais e patate le colture che occupano le prime posizioni secondo i dati fao (2012). II Brasile è il quarto produttore al mondo, con un raccolto stimato per il 2016 di 25 milioni di tonnellate (IBGE, 2015). 
Di grande versatilità, il raccolto è completamente utilizzato, non solo la parte aerea (foglie e steli) ma anche la radice. Le foglie hanno il loro uso in cibo umano (supplemento) e animale (schiacciato). Gli steli, nell'alimentazione animale, sotto forma di silages e fieno e ancora in natura. La radice, nel cibo umano, animale e anche dalle industrie, principalmente farina, amidi, plastiche biodegradabili, prodotti tessili e biocarburanti (CONAB, 2013).

La cultura di Cassava deve affrontare molte sfide, tra cui i problemi fitosanitari (NASCIMENTO JàniOR, 2015). Diverse malattie sono già state segnalate nella cultura, tra loro, marciume radicale, i cui agenti possono essere Lasiodiplodia sp., Pythium sp., Rosellinia sp., Fusarium sp., Neoscytalidium lignicola, Phytophthora drechsleri Tucker tuttavia, gli ultimi tre fitopatogeni sono gli ultimi tre marcire radice nello stato di Alagoas (Figura 1) e nella produzione della maggior parte delle regioni. Le informazioni sul marciume delle radici di manioca sono ancora incipienti ed è di fondamentale importanza per fungere da strumento nel processo decisionale sulla migliore strategia da utilizzare (MASSOLA JR; BEDENDO, 2005; MUNI et al., 2006; NOTARO et al., 2013; SILVA, 2013; FUKUDA, NON voglio ESSERE OTSUBO, 2015).

Tra i microrganismi che causano la radice di manioca marciume $\mathrm{P}$. drechsleri e $F$. solani, sono i più importanti a causa della portata e della gravità causando perdite significative nella produzione. Nei suoli densi e acidificati il verificarsi di Fusarium sp., è più frequente, e una delle caratteristiche di questo patogeno è quello di causare danni allo stelo vicino al suolo da infezione dei vasi xilimici e di conseguenza interferire nella circolazione della linfa, causando così marciume indiretto della radice in qualsiasi causa di sviluppo delle piante. A differenza di Phytophthora, i cui sintomi provocati nelle radici sono di colore marrone e marciume soft-consistency che trasuda un liquido con cattivo odore, già i marciumi di Fusarium sp., senza apparenti disturbi nei tessuti con segni di disidratazione e aspetto mummificato di un marciume secco (MOURA; SILVA, 1997; POLTRONIERI et al., 2001; SILVA, non ho intenzione di TEIXEIRAS, 2012). 
Come misure di controllo del marciume protettivo e radicale, viene utilizzata la gestione del suolo fisico e chimico, ma l'uso di varietà tolleranti associate a pratiche culturali come la rotazione delle colture e il sistema di coltivazione è stato sostenuto positivamente. Nascimento Jonior (2015), sottolinea che è di fondamentale importanza conoscere le condizioni edapho-climatiche della regione coltivata e l'uso di tecniche appropriate che possono essere adottate dall'agricoltore, per avere un ritorno economico all'interno di un sistema agricolo sostenibile. II controllo alternativo dei fitopatogeni con gli antagonisti, in particolare le specie di trichoderma, è già stato studiato, a causa dell'interesse mondiale di utilizzare prodotti meno aggressivi per l'ambiente (SOUSA et al., 2012; SILVA et al., 2014). Per Robbs (1992) e Barbosa; Meza (2009) la competizione per il cibo e l'antibiosi, sono i meccanismi più frequentemente utilizzati da alcune specie di Trichoderma, essendo quindi considerato un eccellente agente di biocontrollo.

In considerazione di quanto sopra, l'obiettivo del presente lavoro era quello di eseguire una revisione bibliografica sui principali fitopatogeni e sul potenziale dell'antagon ismo di Trichoderma coinvolto con marciume della radice di manioca nello stato di Alagoas.

\section{METODOLOGIA}

II presente studio consiste in una revisione della letteratura, condotta tra dicembre 2014 e marzo 2016, in cui è stata effettuata una consultazione ad articoli scientifici, selezionati attraverso la ricerca nel database di Google academic, LILACS, MEDLINE, SciELO, portale di Capes (tesi e tesi), riviste e libri. Dei numerosi articoli correlati, 84 di essi sono stati utilizzati. Come descrittori abbiamo usato: marciume radicale, controllo biologico dei fitopatogeni, fitofollia. 


\section{REVISIONE DELLA LETTERATURA}

\subsection{COLTIVAZIONE DI MANIOCA}

Cassava, una coltura di grande interesse per il nord-est brasiliano, in particolare per lo Stato di Alagoas, è una pianta perenne, arbustiva, appartenente alla famiglia Euphorbiaceus, ricca di amido, utilizzata nel cibo umano e animale o come materia prima per varie industrie (FRAIRE FILHO; BAHIA, 2015).

Nascimento Jànior (2015), sottolinea i vantaggi di questa coltura, grazie alla sua rusticità, a basso costo, adattamento in relazione al clima, al suolo e alle sue caratteristiche nutrizionali che sono legate al grande accumulo di amido nelle sue radici, così come proteine e amminoacidi nelle foglie. È una pianta originaria delle regioni tropicali del Sud America, ma che aveva come luoghi della sua domesticazione Sud-Est asiatico, Africa e America tropicale (NOTARO et al., 2013; SILVA, 2013; FRAIRE SON; BAHIA, 2015).

Nel 2013, 281.718.000 tonnellate di manioca sono state prodotte in tutto il mondo, con il Brasile che è il quarto più grande produttore al mondo con 23.977.757 tonnellate, dietro Nigeria, Indonesia e Thailandia. Le regioni nord e nord-est del Brasile hanno un grande volume di produzione e la superficie coltivata si trova nel sud, nel sud-est e nel midwest, quelle con la più alta resa agricola. Lo Stato di Gusuè è il più grande produttore nazionale di radice di manioca, seguito da Paranào, Maranhào e Bahia (IBGE, 2015).

Ad Alagoas, le regioni che si distinguono come produttori di manioca sono con il $70 \%$ della produzione totale dello Stato (Arapiraca, San Sebastio e Campo Alegre), la zona forestale e la costa settentrionale, essendo la prima regione, quella con il maggior numero di produttori, responsabile di 13.36 tha-1 e 240.448 tonnellate, occupando il secondo posto in termini di produttività rispetto agli altri stati del nord-est (CONAB 2013). 
Notaro (2012) ha sottolineato che questa coltura è ancora agricola familiare e si sviluppa in modo rudimentale, e i principali mezzi di propagazione sono vegetativi e le varietà di bassa qualità genetica sono ancora coltivate. La sua utilità è associata al contenuto di acido cianidrico ( $\mathrm{HCN}$ ) presente nelle foglie e nelle radici, che lo classifica come mite o dolce (<180 mg kg-1 de HCN) noto anche come "macaxeira", utilizzato per il consumo fresco; intermedio (180 - $300 \mathrm{mg} \mathrm{kg}^{-1}$ ) e amaro o selvaggio (> $300 \mathrm{mg} \mathrm{kg-}$ 1) gli ultimi due sono utilizzati per l'alimentazione di animali, amido, farina e biocarburanti (OLIVEIRA et al., 2012).

La più grande utilità della manioca è nell'industria alimentare, principalmente nella produzione di amido destinato al mercato modificato dell'amido, che oltre a servire la cucina regionale in pane, tapioca, beijus, torta di gomma e piede di tomboy; è uno dei prodotti derivati dallo stabilimento che impiega più manodopera e aggiunge un alto valore nel prodotto lavorato, secondo Nascimento Jànior (2015). Per quanto riguarda l'uso per l'estrazione di prodotti fermentati nell'industria dei biocarburanti, la manioca si è dimostrata una buona alternativa nella produzione di etanolo, in quanto presentava un costo energetico inferiore nella produzione, confrontando canna da zucchero e mais, secondo Salla et al., (2010).

Secondo Alves (2006), la fenologia della manioca può variare da 6 a 24 mesi di coltivazione, ma commercialmente è 12 a 18 mesi per la lavorazione di farina e amido e tra 8 e 10 mesi per la tavola. Tra le varietà piantate in Alagoas, spicca rosinha, il cui ciclo varia tra 9 e 10 mesi in terra asciutta, e può essere anticipato per 7-8 mesi in condizioni di sistema irrigate. Questa varietà può raggiungere i tre metri di altezza, mantiene lo stand impianto alla fine del raccolto con una buona produzione di rami e produttività in 21 t ha-1 (DINI et al., 2009).

Nascimento Jànior (2015), riporta nel suo lavoro che anche con la rusticità della coltura è necessaria è attenta alle condizioni adafo-climatiche, affermando che la manioca produce bene in terreni fertili, con $\mathrm{pH}$ tra 5,5 e 7,0 con classe testuale sabbiosa 0 media e buona drenaggio naturale. La condizione climatica ideale è nell'intervallo di temperatura compresa tra i 20 e i 27 gradi centigradi, e può essere stabilita in ambienti 
con temperature comprese tra 16 e 38 o più gradi; con precipitazioni comprese tra 1000 e 1500 mm all'anno-1, in regioni semiaride comprese tra 500 e 700 mm all'anno 1; altitudini da 600 a 800 metri sul livello del mare e un periodo di luce di 12 ore al

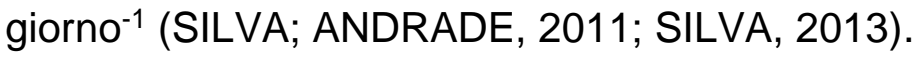

\subsection{MALATTIE NELLA COLTIVAZIONE DELLA MANIOCA}

Diversi fattori possono limitare la produzione di manioca, come l'insorgenza di insetti nocivi e fitopatogeni che causano malattie, compromissione della qualità e dello sviluppo della coltura in Brasile e in tutto il mondo. Secondo Nascimento Jonior (2015), la manioca è suscettibile a diversi fitopatogeni e ciò causa gravi perdite economiche nella produzione.

Tra le malattie importanti spiccano le cause delle lesioni delle foglie come la macchia marrone (Cercosporidium henningsii) macchia bianca (Phaeoramularia manihotis), le foglie che bruciano (Cercospora vicosae), la macchia nera (C. manihobaea) e l'antranosi (Colletotrichum gloeosporioides f.sp. manihotis) che limitano i fotoassimilati (SILVA; ANDRADE, 2011; MORAIS et al., 2013; OLIVEIRA et al., 2013). E marciume radicale, causate da funghi e oomiceti, come Lasiodiplodia sp., Pythium sp., Rosellinia sp., Fusarium sp., Neoscytalidium lignicola, Phytophthora drechsleri Tucker tuttavia, gli ultimi tre fitopatogeni sono gli ultimi tre marcire radice nello stato di Alagoas (Figura 1) e nella maggior parte delle regioni di produzione. Le informazioni sul marciume delle radici di manioca sono ancora incipienti ed è di fondamentale importanza per fungere da strumento nel processo decisionale sulla migliore strategia da utilizzare (MASSOLA JR; BEDENDO, 2005; MUNI et al., 2006; NOTARO et al., 2013; SILVA, 2013; FUKUDA, NON voglio ESSERE OTSUBO, 2015).

Notaro et al., (2013) e Silva (2013) mettono in guardia sugli alti impatti socioeconomici causati dal marciume della radice di manioca negli stati di Maranhào, Paro, Alagoas e Pernambuco, in quanto sta causando perdite significative di produttività, oltre a rendere le aree invivibili per piantare durante i cicli di coltura. Secondo Nascimento Jànior (2015), essendo Omyceto Phytophthora sp e il fungo Fusarium sp., sono i 
principali patogeni della cultura nel nord-est e causano perdite che vanno dal 30 al $70 \%$. L'autore afferma anche che in condizioni favorevoli la malattia può causare una perdita del $100 \%$.

come illustrato nella Figura 1. Aspetto delle colonie e delle strutture riproduttive dei fitopatogeni della manioca che causano isolare nello Stato di Alagoas: Fusarium sp. (A1-A6), Neoscytalidium sp. (B1-B6) e Fitofano sp. (C1-C6). Colonia di Fusarium sp. (A1); conidia (A2); cellule conidiogeniche (A3-A6). Neoscytalidium sp. colonia (B1); Conidia (B2-B6). Colonia fitofano sp (C1); Spomangio (C2); Chlamydospores (C3 e $\mathrm{C} 4)$; Spomangios (C5) e Sporantia in riproduzione (C6).

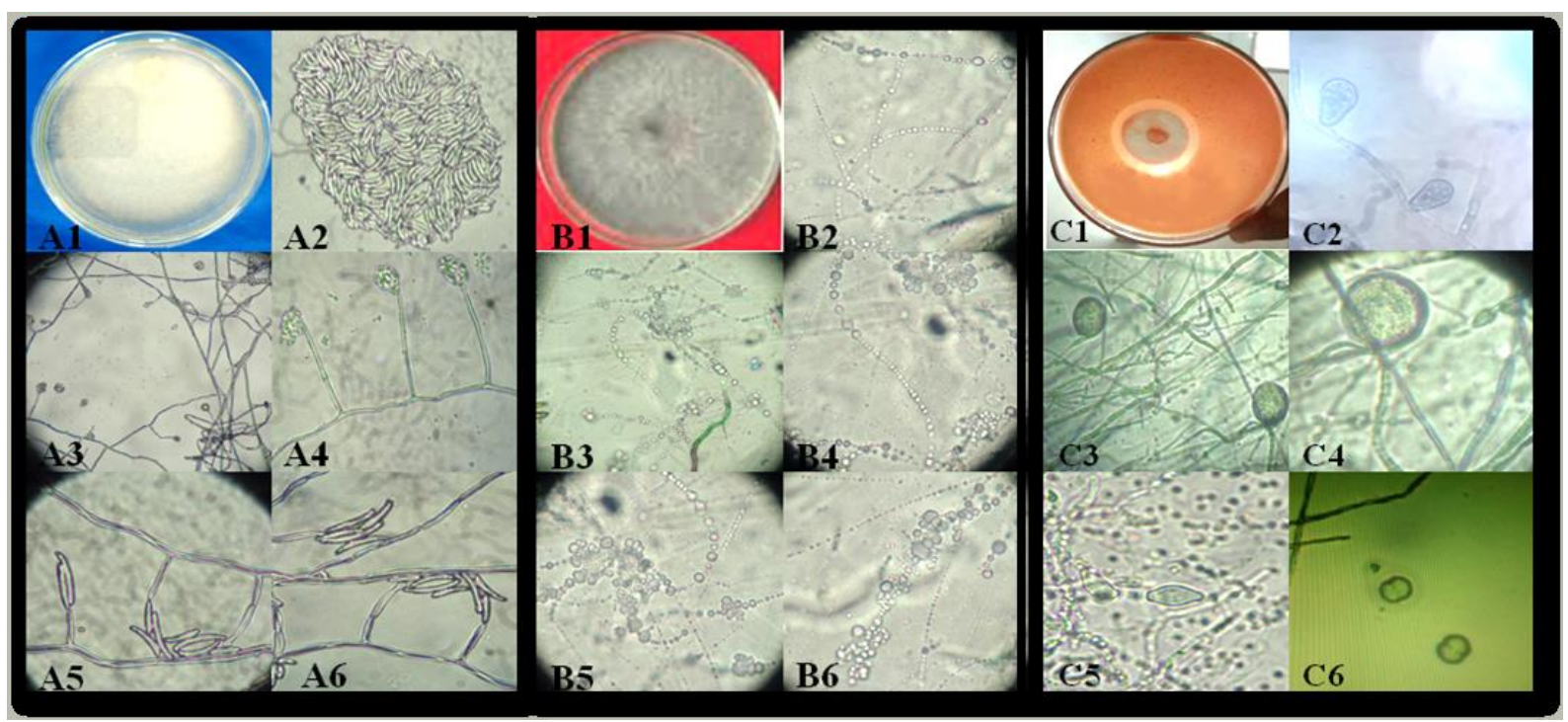

Fonte: SILVA e NASCIMENTO JÚNIOR (2016).

II marciume causato da Fusarium sp., si verifica nelle piante giovani e adulte, con sintomi di appassimento improvviso e grave defogliazione, che può essere potenziata in aree di argilla e terreno scarsamente drenato (SILVA; TEXEIRA, 2012). Questo fatto è già stato segnalato da Gomes; Leal (2003), che quando lavora con la manioca negli stati di Sergipe, Bahia e Alagoas, afferma che il marciume radicale è la malattia più limitante della cultura nella regione nord-orientale, soprattutto quando viene impiantato in aree formate da terreno compattato.

Disponível em: https://www.nucleodoconhecimento.com.br/agronomia-it/antagonismo-di- 
I fitopatogeni che causano il marciume hanno sintomi distinti. Gli autori riferiscono che la fitofano $s p$. di solito attacca le piante adulte, causando marciume "morbido" nelle radici, con odore molto forte, simile a quello della materia organica in decomposizione e colorazione grigiastra. La comparsa di segni è più frequente e visibile nelle radici, ma ci sono rapporti dell'esistenza di giovani che presentano sintomi alla base degli steli delle piante appena gemellate, causando appassimento e morte totale. Nel caso di marciume secco causato da Fusarium sp., si verifica raramente un danno diretto alle radici, ma il problema è accentuato in qualsiasi fase dello sviluppo delle piante. (GOMES; LEAL, 2003; SILVA; TEXEIRA, 2012; NASCIMENTO JÚNIOR, 2015).

\subsection{IL PHYTOPATOGEN FUSARIUM}

II genere Fusarium fu descritto nel 1809 da Link. I rappresentanti di questo genere appartengono al regno Fungi, phylum Ascomycota, classi Eauscomycetes e Pyrenomycetes, ordine Hypocreales, presentando 65 specie, 55 varietà e 22 forme speciali, secondo Wollenweber; Reinking (1935)1 citato da Ventura (1999). È uno dei fitopatogeni più importanti al mondo, con ampia distribuzione geografica, distribuito nel terreno e in associazione con le piante. Avere specie cosmopolite e di altro tipo con eventi limitati a determinati ambienti (VENTURA, 1999). La maggior parte delle specie sono saprobiche, ma ci sono rappresentanti del complesso Fusarium che producono micotossine che possono influenzare la salute umana e animale (URBEN et al. 2009). Seifert (2006); Querales (2010) riferiscono che il genere Fusarium è un gruppo complesso che cresce in numero di specie e genera controversie tassonomiche per i fitopatologi.

Milanesi (2009), commenta che il Fusarium sp., può verificarsi soprattutto nei luoghi di climi tropicali e subtropicali, e ha una grande capacità di sopravvivere per lunghi periodi nel terreno al di fuori dei suoi ospiti, attraverso la formazione di strutture di resistenza chiamate chlamydospores. Tre gruppi all'interno del genere sono gli esempi più specifici di questi cambiamenti, che prendono il nome dal gruppo teleomorfo del complesso Gibberella fujikuroi (Sawada) Wollenw, il Fusarium solani (Mart.) Sacc., e F. graminearum complesso. 
Il genere può presentare colonie di colori che vanno dal viola, purpura scura all'arancione, ma la predominanza è di pallida colonia di rapida crescita, micelio arioso e diffuso (MARTINS, 2005). Puhalla (1981); Martins (2005); Agrios (2005), riferiscono che le strutture di conidia (micro e macro), morfologia, disposizione, varietà di conidiofori e clamofori sono state la base per l'identificazione delle specie Fusarium sp. I tassonomisti classici descrivono la presenza di microconidia unicellulare e unidirezionale e di macroconidia multicellulare frequente con una cellula con un solo nucleo come progenitore geneticamente identico. Le fasi sessuali di Fusarium sono ascomycetes; la spore sessuale è l'ascospore. Quelli assegnati nel genere Nectria hanno ascospores bicellulari.

Ogni cellula dell'ascopore bicellulare non è inucleated ed entrambi i nuclei sono geneticamente identici. Altri, come nel caso di Gibberella, formano ascospore multicellulari. Martins (2005) cita che le caratteristiche morfologiche sono influenzate dall'ambiente e dalla nutrizione, quindi i mezzi di coltura vengono utilizzati per selezionare i microrganismi e raggrupparli, in base alle esigenze nutrizionali che vengono rese disponibili nell'ambiente culturale in condizioni standardizzate per identificarli. A causa di questa plasticità e variazioni nelle caratteristiche fenotipiche presenti in questo fungo, la tassonomia basata solo su concetti morfologici non è affidabile. (MARTINS, 2005; URBENS et al., 2009; QUERALES, 2010).

L'identificazione delle specie di fusarium è uno dei primi passi per il suo studio. Tradizionalmente è stato effettuato sulla base della morfologia, dalla pubblicazione del trattato del genere "Die Fusarien" nel 1935 da Wollenweber; Reinkin91. Sono stati proposti altri sistemi, ma non hanno risolto il problema dell'identificazione delle specie. Gli strumenti per l'identificazione sono stati aumentati e comprendono l'uso di microscopia ottica ed elettronica, mezzi selettivi e differenziali, confronti enzimatici, metaboliti secondari, nonché l'uso di tecnologie immunologiche e molecolari (LEALBERTIOLI, 1998; MARTINS 2005). O'Donnell et al., (2000); Summerell et al., (2003); Ottoni, (2008); commentare che con l'avvento degli strumenti molecolari l'identificazione del Fusarium si basa sulla morfologia, la biologia e le caratteristiche 
della specie, sulla base della compatibilità sessuale, della filologia e delle sequenze delle regioni genomiche.

Per decenni, l'identificazione classica utilizza le caratteristiche morfologiche come base, ma ha generato polemiche, perché diversi tassonomisti e fitopatologi trovano necessario utilizzare altri modelli per separare la specie, mentre altri credono che questi modelli influenzano la tassonomia del genere. È interessante notare che la pratica nella diagnosi di agenti causali di malattie nel piantare l'adozione di modelli genetici sono più adatta agli studi condotti dai micologi. Tuttavia, questi cambiamenti sono rilevanti, poiché in un genere ci possono essere specie molto vicine e questo può portare a diagnosi errate con importanti conseguenze pratiche (QUERALES, 2010).

Secondo Notaro et al. (2013), Fusarium solani Math (Sacc) è la specie che prevalente provoca marciume radicale nella manioca cv branquinha nello stato di Pernambuco. Questa specie produce, in mezzo di coltura, micelio aereo, con colore che vanno dal bianco alla crema, diventando bluastro-marrone quando sono presenti sporodochios. Produce tre tipi di spore asessuate: la macroconidia a forma di slece, con dimensioni approssimative di 15-35 x 4-7 m con 3-5 setti; microconidia, che hanno o non septum, misurano circa 5-10 x 1-3 m e chlamydospores, che sono ialina, globose, liscia o ruvida, misura $6-10 \mathrm{~m}$.

Lo stato sessuale del fungo è Nectria haematococca (Ascomycete). Questo fungo sopravvive su resti culturali infetti, suolo, tuberi e semi, e può persistere per diversi anni. Le condizioni ideali per il vostro stabilimento sono temperature che vanno dai 15 ai 25 gradi centigradi, umidità del $50-75 \%$, presenza di lesioni, piantatura in terreni contaminati e stoccaggio inadeguato. Le lesioni al sistema radicale, in particolare quelle causate dai nematodi, facilitano la penetrazione del fungo nelle radici dei padroni di casa.

\subsection{CONTROLLO DELLE MALATTIE}

Poiché si tratta di un agente patogeno trasportato dal suolo, la sua eliminazione dopo l'installazione nella zona di semina diventa un compito molto difficile e le misure di RC: 55551

Disponível em: https://www.nucleodoconhecimento.com.br/agronomia-it/antagonismo-ditrichoderma 
controllo chimico praticamente non esistono, tranne che per quelle preventive, come I'uso di trattamento delle sementi o di altri materiali di propagazione (LA-AROTTO et al., 2012). II controllo culturale, basato sulla prevenzione delle lesioni durante il raccolto e lo stoccaggio, l'uso di semi sani e substrato privo dell'agente patogeno, utilizzato solo una volta, l'applicazione equilibrata dei nutrienti, l'efficienza nell'irrigazione, così come le varietà che presentano caratteristiche di resistenza e il trattamento di talee (maniva) e substrato con fungicidi per la semina in caso di infezione, può ridurre al minimo le perdite di produzione.

Come misura di controllo, la misura più efficiente si basava sulla ricerca condotta nel nord-est dalla Embrapa Cassava and Fruit And Agricultural Development Company (EMAGRO), è stata l'uso di varietà tollerante (Osso Duro, Cedinha, Bibiana, clone 148/02, Aramaris e Kiriris), associato al sistema di coltivazione e piantatura di rotazione, che ha ridotto il marciume di circa l'80\% (GOMES; LEAL, 2003; BETTIOL, 2009; LOBO J'NIOR et al., 2009; L.A.A. . . . . . . . . . . . . . . . .

Per il controllo di Fusarium sp., il difenochonazolo chimico e il tiram, nel trattamento del suolo e dei semi, sono i più raccomandati a causa dell'efficienza presentata. Tuttavia, il controllo chimico è diventato costante e con l'uso indiscriminato sono sorti problemi ecologici, in quanto questi causano cambiamenti nell'ambiente che favoriscono i fitopatogeni con resistenza dovuta alla pressione selettiva, all'emergere di focolai secondari, alla riduzione della popolazione di microrganismi benefici, a causa del deposito di residui che si accumula nel suolo, nell'acqua e negli alimenti che li rendono dannosi, con effetto deleterio per gli animali e l'uomo (BIZII) , 2015).

In generale, gli elevati costi della pratica del controllo chimico, associati all'emergere di resistenza ai prodotti di uso comune, nonché al divieto del bromuro di metile, rendono essenziale lo sviluppo di tecniche di controllo alternative (BETTIOL, 2009;

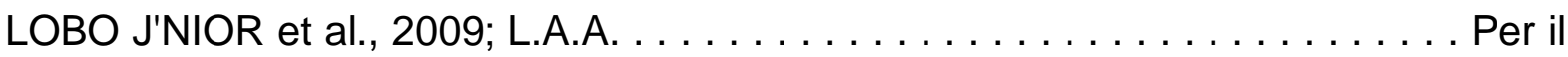
biologico, l'induzione della resistenza alle piante è un'alternativa promettente, in quanto utilizza estratti naturali con proprietà microbiche e/o induttrici (MORAES, 1992; STANGARLIN et al., 2008). 


\subsection{BIOCONTROL DI PHYTOPATOGENI}

II tentativo di ridurre le azioni deleterie di un agente patogeno o di un inoculum è stato una costante in agricoltura, e la sostituzione di prodotti agrochimici con prodotti naturali con soppressione o attività di controllo è stata una delle alternative valide, perché il residuo lasciato è più rapidamente assimilato e scomposto dall'ambiente a causa della sua bassa persistenza (SOU-A, 2013; BICl, 2015).

Ethur, (2006) e Dianese, (2007); relazione sull'importanza della ricerca nell'area del controllo biologico, come l'introduzione di organismi antagonisti in ambienti in cui l'agente patogeno sta causando danni. Cancela (2015), afferma che la dinamica dell'azione di controllo biologico si verifica in modo naturale e consiste nella regolazione del numero di piante e animali da parte dei nemici naturali. Questa strategia è una parte fondamentale dell'equilibrio della natura e di come il controllo chimico abbia vantaggi e svantaggi. Tra i vantaggi è il fatto che si tratta di una misura non tossica, non causa squilibrio, non ha controindicazioni, fornisce un controllo esteso ed efficiente quando non c'è controllo chimico, perché avrà bisogno di più tecnologia a causa del suo effetto lento, difficile da acquisire e non può sempre essere applicato in qualsiasi momento dell'anno.

Mantenere l'equilibrio nell'Agroecosistema è una pratica di controllo biologico in modo che il sistema patogeno non causi danni significativi nell'azione biocontrollo degli organismi non patogeni del sistema. Gli autori riferiscono anche che la malattia è più di una relazione di interazione patogeno/ospite, perché l'influenza dell'ambiente e anche una diversità di organismi non patogeni riposa nel sito di infezione può limitare o aumentare l'aggressività del patogeno, o resistenza all'ospite. Così, patogeno, ospite e antagonista interagiscono tra loro nei componenti del controllo biologico (BII, 2015; CANCELS 2015).

Un fattore limitante per l'uso di prodotti biologici è la disponibilità sul mercato per questa modalità di controllo. Un altro problema è che molti prodotti naturali non sono adeguatamente registrati per l'uso su scala commerciale (SILVA et al., 2008). La 
presenza del fitopatogeno interferisce con l'omeostasi vegetale, ma può anche agire attivando meccanismi di difesa e/o attivando geni che conferiscono resistenza alla pianta ospite (AGRIOS, 2005). Michereff et al., (1993), afferma che l'agente patogeno ha determinante attività che coinvolgono la crescita, l'infettività, l'aggressività, la virulenza e altre attribuzioni dell'agente patogeno o dei processi, che determinano l'infezione, lo sviluppo dei sintomi e la riproduzione. L'interazione tra antagonista, ospite e agente patogeno non si verifica in un modo particolare, ma reciprocamente.

Diversi autori hanno già descritto la capacità dei biocontranger svincolare e interferire nello sviluppo del fitopatogene mediante meccanismi di azione come la competizione per lo spazio e i nutrienti all'antibiosi, il micoparassitismo o l'iperparassitismo, la predazione, l'induzione della resistenza, aumentando così il vantaggio competitivo nell'ambiente. Questi meccanismi variano da specie a specie e anche da lignaggio a lignaggio all'interno della stessa specie, secondo l'interazione ospite-parassita. (CARVALHO, 2006; BETTIOL; MORANDI, 2009; BRITO et al., 2010; ZUCCHI, 2010; DIAS, 2011; MACHADO et al., 2012; BIZI, 2015; CANCELA, 2015; FIPKE, et al., 2015).

Molti microrganismi hanno presentato un alto potenziale biocontrollore e il genere Trichoderma Pers. è stato utilizzato in diversi studi. T. hamatum (Bonord.) Bainier, T. viride Pers., T. aureoviride Rifai, T. harzianum Rifai, T. koningi Oudem, T. pseudokoningii Rifai e T. longibrachiatum Rifai sono i più citati (BETTIOL; GHINI, 2005). Ezziyyani et al., (2007) riferiscono che la frequenza di Trichoderma spp., è dovuta alla sua versatilità sia per concorrenza, iperparassitismo e antibiosi a fitopatogeni come Phytophthora, Fusarium e Neoscytalidium. Remuska \& Pria (2007) valutando l'effetto antagonistico di Trichoderma spp., nel controllo della crescita miceliale dei funghi fitopatogeni ha osservato che questo esercitava antagonismo su alcuni fitopatogeni della radice, come lo Sclerotium rolfsii, l'aphanidermatom Pythium e il Fusarium solani, quest'ultimo genere descritto come uno dei principali fitopatogeni associati al marciume della radice di Cassava nello stato di Pernam. 
Hoffmann et al., (2015) ha riferito di avere l'80\% del controllo di Fusarium sp., utilizzando gli isolati spp Trichoderma. Da Silva et al., (2011); Pereira (2013) ha dimostrato nelle sue opere un grande livello di inibizione della crescita micelica di $F$. solani da specie trichoderma spp. Souza et al., (2015) ha avuto anche risultati promettenti con Fusarium sp., e Bipolaris sp., utilizzando lo stesso biocontrollore. Altri studi di Bomfim et al., (2010); Dias (2011); Lazarotto et al., (2012); Santos et al., (2012); Marques et al., (2014), ha ottenuto risultati promettenti presentando prestazioni buone o eccellenti, per quanto riguarda l'inibizione della crescita miceliale dei fitopatogeni testati da loro.

\subsection{STRATEGIE DI BIOCONTROLLO DEL TRICHODERMA}

Il genere Trichoderma, appartenente al Regno dei Funghi, phylum Ascomycota, classi Eauscomycetes e Pyrenomycetes, ordine Hypocreales, famiglia Hypocreaceae, genere Hypocrea (teleomorph). Questo fungo ha un grande potenziale antagonistico ed è spesso associato a terreni soppressivi. (MELO, 1991). Secondo Harman et al. (2004); Harman (2005), il suo utilizzo può offrire altri vantaggi: decomposizione della materia organica, concorrenza con una microflora deleteria attraverso la colonizzazione della rizosfera, produzione di antibiosi e induzione di resistenza.

Le specie di trichodema sono già state documentate per il controllo di vari patogeni, come Rhizoctonia solani K'hn, Sclerotium rolfsii Sacc., Sclerotina sclerotiorum (Lib.) di Bary, Fusarium spp. e Pythium spp. (MELO; AZEVEDO, 1998). Numerose specie di Trichoderma, tra cui T. harzianum Hifai, hanno mostrato un alto tasso di parassitismo e attività antagonista per fitopatogeni come S. rolfsii, S. minor e S. sclerotiorum, R. solani e Pyricularia grisea Sacc. (ROLLAN et al., 1999; RONDÓN et al., 2007).

Secondo Dos Santos (2010), l'isolato di T. harzianum ha presentato in vitro l'antagonismo sul fungo S. rolfsii; essere in grado di iperparassionizzare le ife del patogeno e competere per lo spazio e nutriente. Silva et al. (2013), lavorando con isolati di Trichodema spp., come agente di controllo biologico di S. lignicola, ha osservato che inizialmente l'isolato di Neoscytalidium sp. era molto promettente 
quando si confrontava con Trichoderma spp., ma quando sono stati trovati nel piatto di Petri, Trichoderma spp., sovrapposto la colonia patogena. Osservando la regione in cui l'antagonista si sovrappone ai fitopatogeni, è stato possibile notare una degradazione delle ife del patogeno per azione enzimatica, poiché si distingueva accuratamente solo spore e ifesi dall'antagonista.

Per Robbs (1992) e Barbosa; Meza (2009) la concorrenza per il cibo e l'antibiosi, sono i meccanismi più frequentemente utilizzati dagli agenti di biocontrollo, poiché la riproduzione e la colonizzazione rapida sono attributi degli organismi che utilizzano le risorse disponibili in modo più efficiente e rapido, garantendo la loro sopravvivenza. Secondo Ethur (2006), la competizione è una delle principali strategie di trichoderma $s p$. isola, per questo fatto vengono utilizzati come biocontrollori.

La secrezione di sostanze antibiotiche organiche (antibiosi), prodotte da microrganismi, che anche a bassa concentrazione interferiscono nelle attività metaboliche, l'inibizione o l'arresto della crescita e della sporulation, la riduzione della germinazione delle spore e le distorsioni delle ife ed endolisi, specifiche o di ampio aspetto, sono dispositivi importanti per l'efficienza degli organismi antagonici (LOBO JUNIOR; ABREU, 2000; BOMFIM, 2007; SOU-A, 2013). Secondo Claydon et al., (1987), gli antibiotici sono prodotti del metabolismo secondario dei loro produttori e possono essere più importanti nell'inibire altri organismi oltre alla concorrenza per le sostanze nutritive. L'antibiosi è stata segnalata anche da diversi ricercatori come uno dei meccanismi di azione delle specie di Trichoderma (BETTIOL, 1991; DIAS, 2011; CARVALHO FILHO, 2013). Gliotoxin, viridee e trichodermine sono alcune delle sostanze prodotte dalla specie Trichoderma in grado di inibire lo sviluppo di altri funghi (Amorim et al., 2011). Ethur (2006) ha riferito che la gliotossina era responsabile dell'inibizione della germinazione degli spomangio e della crescita micelia de/ Pythium ultimum.

Secondo Bomfim et al., (2010) e Souza (2013), il biocontrollore avrà successo se sarà in grado di degradare la parete cellulare (composta da carboidrati, come la chitina), così come proteine e lipidi in quantità significative del fitopatogeno $\mathrm{o}$ interrompere $\mathrm{i}$ 
processi relativi alla sua sintesi. Pertanto, la produzione di enzimi che idrolizzano i componenti della parete cellulare dei fitopatogeni, in particolare le chitinasi e le glucanasi, è una caratteristica comune in molti agenti di biocontrollo. Alcuni rappresentanti del genero Trichoderma sono citati in opere come un eccellente produttore di cellulasi e chitinasi, enzimi che degradano cellulosa e chitina (MELO, 1991; HARMAN et al., 2004).

II micoparassitismo, una situazione in cui il microrganismo antagonista vive all'interno o all'interno del fungo antagonizzato, alimentandosi e sviluppandosi a sue spese, è un altro importante meccanismo d'azione come attributo di un buon biocontrollore. II micoparassitismo comporta antibiosi e cannibalismo causato dall'azione di enzimi idrolitici, come chitinasi, glucanasi, proteasi e lipasi, che causano la morte di uno di essi che serve così come cibo per il sopravvissuto (LOBO JUNIOR; ABREU, 2000; HARMAN, 2000; BOMFIM, 2007; BARRA et al., 2008; (2010).

Le chitinasi e i z-1,3-glucanasi sono direttamente coinvolti nelle interazioni del micoparassitismo tra la specie di Trichoderma e i loro ospiti, a causa della maggior parte dei funghi fitopatogeni che possiedono nella loro parete cellulare chitina, organizzati in strati regolarmente ordinati e - 1,3-glucani disposti come riempimento in modo amorfo. La chitinasi agisce in modo casuale e degrada le strutture biologiche e i componenti delle cellule fungine, rilasciando oligomeri che inducono esoquitinasi, iniziando così l'attacco micoparassitico, idrolmentecolando le oligosacharides della parete cellulare del fitopatogeno. (GIESE et al., 2003; MARCELLO, 2008; BAUERMEISTER et al., 2010).

Melo (1996) riferisce che il micropocrisismo necrotrofisico è efficiente nell'antagonismo dei fitopatogeni, in particolare quelli con strutture di resistenza considerate difficili da attaccare da microrganismi, come spros, sclerotia, chlamydospores e microsclerotia. Melo, melo. Azevedo (1998) afferma che Trichoderma sp. ha caratteristiche micoparassiti, perché possono individuare e rilevare i fitopatogeni, possibilmente da stimoli biochimici, crescono verso l'hyphae dei funghi suscettibili, e quando entrano in contatto, formano strutture come gli appressolatori, si arricciano, nella loro piena 
estensione, li penetrano e li digeriscono. II genere Trichoderma è senza dubbio un eccellente agente di controllo microbico, perché presenta, oltre alle competenze sopra descritte, caratteristiche essenziali, essendo: impatto ambientale e basso o nullo, facile propagazione (SPIEGEL; CHET, 1998), capacità di sopravvivere in ambienti sfavorevoli, oltre a contenere popolazioni di patogeni che vivono nel suolo (VINALE et al., 2008).

\section{CONSIDERAZIONI FINALI}

Le relazioni ecologiche interspecifiche sono eventi che si verificano a vari livelli in natura e sono per lo più fatti di forme dimmoniche, nella condizione di rapporto tra microrganismi che sono la concorrenza, l'antibiosi e il parassitismo il più frequente. Così i microrganismi che causano malattie nelle piante non solo causano perdite economiche, ma si evolvono simultaneamente, causando così danni dalla fase iniziale dello sviluppo delle colture. Sono stati utilizzati metodi tradizionali di controllo dei fitopatogeni, l'applicazione di pesticidi chimici e fungicidi, è stata anche una forma molto frequente che causa gravi problemi dovuti agli impatti ambientali.

Uno dei modi più promettenti per ottenere una riduzione nell'uso di queste sostanze agrochimiche, o anche una futura sostituzione, controllo biologico attraverso i numerosi biocontrollori come i funghi del genere Trichoderma che sono efficienti e non causano impatti ambientali distruttivi.

\section{RIFERIMENTI}

AGRIOS, G.N. Plant pathology. Amsterdam : Elsevier Academic Press. 5.ed, 2005. $922 p$.

ALVES, A., A., C.. Fisiologia da mandioca. In: Aspectos socioeconômicos e agronômicos da mandioca. EMBRAPA Mandioca e Fruticultura Tropical, Cruz das Almas, 2006., 817p. 
AMORIM, L.; REZENDE, J. A. M.; BERGAMIN FILHO, A. Manual de Fitopatologia. Piracicaba: Agronômica Ceres. v. 1,4. Ed, p. 383 -387, 2011.

BARBOSA, R.J.F.; MEZA, C.L.S. Antagonismo in vitro de Trichoderma harzianum rifai sobre Fusarium oxysporum schlecht $f$. sp passiflorae en maracuyá (Passiflora edulis sims var. flavicarpa) del municipio zona bananera colombiana. Rev. Fac. Nal. Agr. Medellín, v.62, n.1, p.4743-4748, 2009.

BARRA, V. R.; ROMEIRO, R.S.; FERRAZ, H.G.M.; MACAGNAN, D.; SILVA, H. S. A.; MOURA, A. B.; HALFELD-VIEIRA, B. A; MENDONÇA, H. L.; VIEIRA JÚNIOR, J. R. Potencialidade antagonística em alguns procariotas agentes de biocontrole de enfermidades de plantas. Ver. Summa Phytopathologica, v.34, n.2, p.121-126, 2008.

BAUERMEISTER, A.; REZENDE, M. I.; GIESE, E. C.; DEKKER, R. F. H.; BARBOSA, A. M. 1,3-Glucanases Fúngicas: produção e aplicações biotecnológicas. LONDRINA SP. rev. Semina: Ciências Exatas e Tecnológicas, , v. 31, n. 2, p. 75-86, jul./dez. 2010. http://www.uel.br/seer/index.php/semexatas/article/view/5249

BETTIOL, W. Controle biológico de doencas do filoplano. In: BETTIOL, W., org. Controle biológico de doencas de plantas. Jaguariuna: EMBRAPA-CNPDA, 1991. p.33-52 (EMBRAPA-CNPDA. Documentos, 15).

BETTIOL, W.: MORANDI, M.A.B. Controle Biológico de Doenças de Plantas no Brasil. In: BETTIOL, W.: MORANDI, M.A.B. (Org). Biocontrole de Doenças de Plantas: uso e perspectivas. Jaguariúna - SP: Embrapa Meio Ambiente, p. 7-14, 2009.

BETTIOL, W.; GHINI, R. Solos Supressivos. In: MICHEREFF,S. J.; ANDRADE, D. E. G. T.; MENEZES, M. (Eds.) Ecologia e Manejo de patógenos radiculares em solos tropicais. Recife:UFRPE, Imprensa Universitária, 2005. cap.6, p. 125-143.

BIZI, R. M., Utilização de fungos no Controle Biológico de Doenças. < http://www.floresta.ufpr.br/alias/lpf/public_html/contbio03.html.> Acesso em 16/11/2015. 
BOMFIM, M. P., Antagonismo in vitro e in vivo de Trichoderma spp. a Rhizopus stolonifer em maracujazeiro amarelo. Vitória da Conquista BA, 2007. 74f.: Dissertação de Mestrado. Universidade Estadual do Sudoeste da Bahia. 2007

BOMFIM, M. P., SÃO JOSÉ, A. R., REBOUÇAS, T. N. H., ALMEIDA, S. S. D., SOUZA, I. V. B., \& DIAS, N. O . Avaliação antagônica in vitro e in vivo de Trichoderma spp. a Rhizopusstolonifer em maracujazeiro amarelo. Summa Phytopathologyca, v.36, n.1, p. 61-67, 2010.

BRITO, F. S.; MILLER, P. R. M., STADNIK, M. Presença de Trichoderma spp em composto e suas características para o controle de fitopatógenos. Revista. Bras. de Agroecologia, Porto Alegre , p. 43-53. 2010.

CANCELA, K. C., Controle Biológico Florestal Conceitos, Terminologia, Descrição e Caracterização de Agente. Proteção florestal Disponível em $<$ http://www.floresta.ufpr.br/alias/pf/public_html/contbio01.html> Acesso em 16/11/2015.

CARVALHO FILHO, M. R., Relações filogenéticas, identificação e potencial de uso de isolados de Trichoderma no controle do mofo branco e como promotores de crescimento do feijoeiro. Brasília-DF, 2013. 123f. Tese de Doutorado. Programa de Pós-graduação em Fitopatologia, Universidade de Brasília. Brasília. 2013.

CARVALHO, A. C., Bioprospecção de isolados de Trichoderma stromaticum para o controle biológico da Vassoura-de-Bruxa do Cacaueiro. 2006. 82f. Dissertação de Mestrado. Universidade Estadual de Santa Cruz. 2006.

CONAB- CLAYDON, N.; ALLAN, M.; ITANSON, J.R.; AVENT, A. G. Antifungal alkyl pyrones of Trichoderma harzianum. Transactions of the British Mycological Society, v.88, p.503-513, 1987. COMPANHIA NACIONAL DE ABASTECIMENTO. Perspectivas para a agropecuária. Conab, Brasília , v.1, 2013., 154p. 
DA SILVA, D. C. V., TIAGO, P. V., \& DE SOUZA-MOTTA, C. M. . Isolamento e seleção de fungos filamentosos do solo de sistemas agroflorestais do Município de Bom Jardim (PE) com base na capacidade de produção de enzimas hidrolíticas1. Revista Brasil. Bot, 34(4), 607-610. 2011

DIANESE, A. de C. Variabilidade e controle de Phytophthora palmivora (Podridão-do-pé) e controle da varíola (Aperisporium caricae) do mamoeiro (Carica papaya). 2007. 109f. Tese Doutorado - Universidade de Brasília, Brasília. 2007

DIAS, P. P., Controle biológico de fitopatógenos de solo por meio de isolados de fungos do gênero Trichoderma e sua contribuição no crescimento de plantas. 2011. 101 f. Tese Doutorado. Universidade Federal Rural do Rio de Janeiro. Seropédica - Rio de Janeiro. 2011

DINIZ, M. de S.. Efeito da manipueira na adubação da mandioca. Revista Raízes e Amidos Tropicais, v. 5, 2009.

DOS SANTOS, J. et al. Atividade hiperparasíticade Trichoderma spp. sobre escleródios de Sclerotinia sclerotiorum em solo. In: SEMINÁRIO DE INICIAÇÃO CIENTÍFICA TECNOLÓGICA,7,2010.Anais...EPAMIG,2010.Online.Disponívelem:http://www.epa mig.br/index.phpoption=com_docman\&task=cat_view\&gid=121\&dir=DESC\&order=da te\&limit=10\&limitstart=10.Acesso em Dezembro 2015

ETHUR, L.Z. Dinâmica populacional e ação de Trichoderma no controle de fusariose em mudas de tomateiro e pepino. 2006. 154p. Tese (Doutorado em Fitopatologia) - Universidade Federal de Santa Maria, Santa Maria-RS. 2006.

EZZIYYANI, M.; REQUENA, M. E.; EGEA-GILABERT, C.; CANDELA, M. E. Biological Control of Phytophthora Root Rot of Pepper Using Trichoderma harzianum and Streptomyces rochei in Combination. Journal of Phytopathology , [S.I], v.155, n.6, p. 342-349, 2007 
FAO. Faostatidtics Database. Disponivel em <https://www.fao.org.br/FAO_Brf2mpu3a.asp> Acesso em Dezembro de 2015.

FIPKE, G. M; PAZINI, J.B.; ETHU,L. Z. Antagonismo de isolados de Trichoderma spp. ao Sclerotinia sclerotiorum em diferentes temperaturas. Magistra, Cruz das Almas BA, V. 27, N.1, p. 23 - 32, Jan./Mar. 2015

FUKUDA C.; OTSUBO A.A. Cultivo da mandioca na região centro sul do Brasil. Embrapa Mandioca e Fruticultura. HTTP://sistemasdeproducao.cnptia.embrapa.br/FontesHTML/Mandioca/mandioca_c entrosul/doenc s.htm . Acessado em 16 de julho de 2015.

FRAIFE FILHO G. A., BAHIA J. J. S., Mandioca. Ceplac-Cepec-BA. http://www.ceplac.gov.br/radar/mandioca.htm . Acessado Dezembro de 2015.

GIESE, E. C.; BARBOSA, A. M.; SILVA M.L.C. Glucanases Fúngica: Produção e aplicações das -1,3 e -1,6 glucanases. Revista Biotecnologia Ciência e Desenvolvimento, Brasília-DF, № 30 , P.97-104.. jan/jun 2003.

GOMES, J. C.; LEAL, E. C., Cultivo da Mandioca para a Região dos Tabuleiros Costeiros. In.: Podridão Radicular. Embrapa Mandioca e Fruticultura. Sistemas de Produção, 11. ISSN 1678-8796 Versão eletrônica/Jan/2003. https://sistemasdeproducao.cnptia.embrapa.br/FontesHTML/Mandioca/mandioca_ta bcosteiros/doencas.htm . Acesso 13/12/2015.

HOFFMANN, C. A. et al.. Potencial de antagonismo de isolados de Trichoderma sp. contra o isolados de Fusarium sp., in vitro. Revista Verde de Agroecologia e Desenvolvimento Sustentável, v. 10, n. 1, p. 236-242, 2015.

HARMAN, G. E. The Nature and Application of Biocontrol Microbes II: Trichoderma spp. Overview of Mechanisms and Uses of Trichoderma spp. Phytopathology, St Paul, v. 96, p. 190-194. 2005 
HARMAN, G. E. Myth and dogmas of biocontrol changes in perceptions derived from research on Trichoderma harzianum T-22. Plant Disease, St. Paul, v. 84, p.377-393, 2000.

HARMAN, G.E. HOWELL, C. R., VITERBO, A., CHET, I., \& LORITO, M. Trichoderma species-opportunistic, avirulent plant symbionts. Nature Reviews Microbiology. V.2, p. 43-56. 2004.

IBGE - Instituto Brasileiro de Geografia e estatística. PAS - Pesquisa Anual de Serviços, 2015. [online]Disponível na internet via WWW URL: http://www.ibge.gov.br/home/. Arquivo consultado Dezembro de 2015.

LAZAROTTO, M.; BOVOLINI, M. P.; MACIEL, C. G.; MUNIZ, M. F. B. Seleção in vitro de Isolados de Trichoderma spp. com Potencial de Antagonismo a Isolados Patogênicos de Fusarium spp. In: XVI Simpósio De Ensino, Pesquisa E Extensão, Unifra, v. 3, 2012.

LEAL- BERTIOLI, S. C. DE M. O enfoque molecular na sistemática de fungos. Revisão Anual de Patologia de Plantas. V.6. p. 197-230, 1998

LOBO JÚNIOR, M. e ABREU, M.S. Inibição do crescimento micelial de Sclerotinia sclerotiorum por metabólitos voláteis produzidos por alguns antagonistas em diferentes temperaturas e pH's. Ciência Agrotécnica, Lavras, v.24, p.521-526, 2000.

LOBO JUNIOR, M.; BRANDÃO, R. S.; CORRÊA, C. A.; GÖRGEN, C. A.; CIVARDI, E. A.; OLIVEIRA, P. de. Uso de braquiárias para o manejo de doenças causadas por patógenos habitantes do solo.Santo Antônio de Goiás: Embrapa Arroz e Feijão, 2009. 8 p. (Embrapa Arroz e Feijão. Comunicado técnico, 183).

MACHADO, D. F. M.; PARZIANELLO, F. R.; SILVA, A. C.F. e ANTONIOLLI, Z. I. Trichoderma no Brasil: o fungo e o bioagente. Revista de Ciências Agrárias, v.35, n.1, p. 274-288, 2012. 
MARCELLO, C. M. Avaliação da expressão e caracterização de uma exo-b-1,3glucanase envolvida no mecanismo de micoparasitismo de Trichoderma asperellum. Brasília-DF, 2008. 89f. Tese de Doutorado. Laboratório de Enzimologia Departamento de Biologia Celular do Instituto de Biologia da Universidade de Brasília - UnB. Brasília - DF. 2008.

MARQUES, H. I. P.; SILVA, M. B.; MARQUES, M. D. P.; RODRIGUES, R. C.; RIBEIRO, P. R. C. C. Inibição do crescimento micelial de Trichoderma harzianum por fertilizantes líquidos. Enciclopédia Biosfera, v. 10, n. 18, p. 2040, 2014.

MARTINS, M. K.. Variabilidade genética de isolados de Fusarium spp. e estudo da interação com a planta hospedeira. 2005. 110f. Diss. Tese (Doutorado em Agronomia)-Escola Superior de Agricultura" Luiz de Queiroz", Universidade de São Paulo, SP.[Links], 2005

MASSOLA JR, N.S.; BEDENDO, I. P.. Doenças da mandioca. In: KIMATI, H. et al. Manual de fitopatologia: Doenças das plantas cultivadas;. 4" Ed.vol. 2, p. 340-341 São Paulo: Agronômica Ceres, 2005

MELO, I. S. Potencialidades da utilização de Trichoderma spp. no controle biológico de doenças de plantas. In: BETTIOL, W. (Ed.). Controle biológico de doenças de plantas. Jaguariúna: CNPDA/EMBRAPA, 1991. p. 135-156.

MELO, I. S.; AZEVEDO, J. L. Ecologia Microbiana. Jaguariúna: Embrapa DNPMA, p. 393-419, 1998.

MELO, I.S. Trichoderma e Gliocladium como bioprotetores de plantas. Revis. Anu. Patol. Plantas v. 4, p. 261-295. 1996.

MENEZES, J. P., LUPATINI, M., ANTONIOLLI, Z. I., BLUME, E., JUNGES, E., \& MANZONI, C. G. Variabilidade genética na região its do rDNA de isolados de trichoderma spp.(Biocontrolador) e Fusarium oxysporum f. sp. Chrysanthemi. Ciência e Agrotecnologia, 34(1), 132-139.2010 
MICHEREFF, S.J.; MENEZES, M.; MARIANO, R.L.R. Potencial de Trichoderma para o controle da antracnose do sorgo. Fitopatologia Brasileira, Brasília, v. 18, p. 392-398, 1993.

MILANESI, P.M. Caracterização, toxicidade e patogenicidade de Fusarium spp. em genótipos de soja em sistema plantio direto. 2009. 91 p. Dissertação (Mestrado em Agronomia) - Universidade Federal de Santa Maria, Santa Maria, 2009.

MORAES, W. B. C. Controle alternativo de fitopatógenos. Pesquisa Agropecuária Brasileira, v. 27, n. 13, p. 175-190, 1992.

MORAIS, M. dos S.; NASCIMENTO, L. C do; MOREIRA, K. A.; SILVA, M. da; CAVALCANTI, N. T. D. O. Levantamento e avaliação da incidência das doenças da mandioca no estado da Paraíba. Summa Phytopathologica, v. 39, n. 3, p. 204. 2013.

MOURA, G. de M.; SILVA, M. D. O. da. Avaliação de resistência de cultivares de mandioca à podridão de raízes. Embrapa-CPAF/AC. Comunicado Técnico, 4p. Rio Branco 1997.

MUNIZ J. N, RUFFINO-NETTO A, YAMAMURA M, VILLA TCS, ARCENCIO R. Aspectos epidemiológicos da coinfecção tuberculose e vírus da imunodeficiência humana em Ribeirão Preto (SP), de 1998 a 2003. J. Bras. Pneumol., 32(6):529-34, 2006

NASCIMENTO JÚNIOR, N. A. Efeito da casca de mandioca no controle da podridão radicular causada por Phytophthora sp. em mandioca de mesa (Manihot esculenta Crantz) var. Rosinha em ambiente irrigado. Rio LargoAL,2015.82f. Tese de Doutorado. (Programa de Pós-Graduação em Proteção de Plantas da Universidade Federal de Alagoas. Alagoas. 2015. 
NOTARO, K. A. MEDEIROS, E. V., SILVA, C. A. D., \& Barros, J. A. Prospecção de fitopatógenos associados á podridão radicular da mandioca em Pernambuco, Brasil. Biosci. J., Uberlândia, v. 29, n. 5, p. 1832-1839, 2013.

NOTARO, K. A. Prospecção de fitopatógenos e caracterização de solos arenosos envolvidos na supressividade ou conducividade da podridão radicular da mandioca, causada por Neoscytalidium lignicola/ Krystal de Alcantara Notaro. Garanhuns-PE, 2012. 111f. Dissertação (PROGRAMA DE PÓS-GRADUAÇÃO EM PRODUÇÃO AGRíCOLA). Universidade Federal Rural de Pernambuco - Unidade Acadêmica de Garanhuns, 2012.

O`DONNELL, K.; NIRENBERG, H.I.; AOKI, T.; CIGELNIK, E. A multigene phylogeny of the Gibberella fujikoroi species complex: detection of additional phylogenetically distinct species.Mycoscience, Tokyo, v. 41, p. 61-78, 2000.

OLIVEIRA, L. G. SILVA, A.C.S; SILVA, R. L. ,LIMA, D. W. S \& LIMA, G; COSTA. Atividade proteolítica de fungos de solo fitopatogênicos ao Feijão-Caupi [Vigna unguiculata (L.) Walp.] In: III CONAC Congresso Nacional de Feijão-Caupi. Pernambuco. Resumo expandido. 4p. 22-24 de abril Recife Pernambuco 2013.

OLIVEIRA, N. T; J. M. A., UCHÔA, S. C. P., RODRIGUES, G. S., MELVILLE, C. C., \& de ALBUQUERQUE, J. D. A. A.. Caracterização e identificação de clones de mandioca produzidas em Roraima para o consumo in natura. Revista Agro@mbiente On-line, v. 5, n. 3, p. 188-193, 2012.

OTTONI, R.J. Analise da incidência de Fusarium spp. toxigenicos e de níveis de fumonisinas em grãos ardidos de milho hibrido. 2008. 54 p. Dissertação (Mestrado em Microbiologia Agrícola) - Escola Superior de Agricultura "Luiz de Queiroz", Universidade de São Paulo, Piracicaba, 2008.

PEREIRA, C O.F. Estudo da patogenicidade e controle biológico de Fusarium sp. Com Trichoderma sp. 2013.74f. Dissertação. Universidade de Caxias do Sul. 2013. 
PUHALLA, J.E. Genetic considerations of the genus Fusarium. In: Nelson, P.E.; Toussoun, T.A.; Cook, R.J. (Ed) Fusarium: diseases, biology, and taxonomy. Pennsylvania: Pennsylvania State University, v.27, p.291-305, 1981.

QUERALES, P. J.. Caracterização morfológica e genética de Fusarium spp. isolados de sementes e associados à podridão do colmo de milho (Zea mays L.). Diss. Escola Superior de Agricultura "Luiz de Queiroz, 2010.

REMUSKA, A.C.; DELLA PRIA, M. Efeito de Bacillus truringiensis e Trichoderma sp. no crescimento de fungos fitopatogênicos. Publication UEPG: Ciências Exatas e da Terra, Agrárias e Engenharia, Ponta Grossa, v.13, n.3, p.31-36, 2007.

ROBBS, F. C. Controle Biológico de doenças em plantas. In: NETO, A. M. A.; BARAN, C. L. (ed.). Manual de Controle Biológico. Rio de Janeiro: Lidador. 1992. p.4651.

ROLLAN, MONACO C, NICO A. Efecto de la tempratura sobre la interaccion invitr o entre especies de Tricihoderma y Sclerotinia sclerotiorum, S. minor y Sclerotium rolfsii. Invest. Agr.: Prod. Prot. Veg. 14:1-16. 1999.

RONDÓN T.R, GUTIÉRREZ GR, ZAYAS A.D.P, PÉREZ LA, CUTIÑO Y.L. Efectividad in vitro de Trichoderma harzianum Rifai para el biocontrol de Rhizoctonia solani Kühn y Pyricularia Grisea sacc. aislados en el cultivo del arroz (Oryza sativa I.). Fitosanidad 11: 29-34.2007.

SALLA, D. A., CABELLO, C. Análise energética de sistemas de produção de etanol de mandioca, cana-de-açúcar e milho. Revista Energia na Agricultura. Botucatu, vol. 25, n.2, p.32-53., 2010.

SANTOS, C. C., OLIVEIRA, F. A. de; SANTOS, M. S. dos; TALAMINI, V.; FERREIRA, J. M. S.; SANTOS, F. J. dos., Influência de Trichoderma spp. sobre o crescimento micelial de Thielaviopsis paradoxa. Scientia Plena 8, 047309 www.scientiaplena.org.br . 2012 
SEIFERT, K.A. Enhancing the relevance of fungal taxonomy to plant pathology: phylogenetics, molecular diagnostics, and long-term memory1. Symposium contribution. Canadian Journal of Plant Pathology, Otawa, v. 28, p. S280-S287, 2006.

SILVA, A. N.; AZEVEDO, G. B.; ROCHA-SOBRINHO, G. G.; NOVAES, Q. S.; Efeito de produtos químicos e de Trichoderma spp. no controle de Fusarium solani do maracujazeiro. Interciencia, v. 6, p. 398-403, 2014.

SILVA, C. A. D., DE MEDEIROS, E. V., BEZERRA, C. B., DE MORAIS; SILVA, W., DE BARROS, J. A., \& DOS SANTOS, U. J. Interferência da incorporação de matéria orgânica no solo no controle da podridão negra da mandioca, causada por Scytalidium lignicola. Biosci. J., Uberlândia, v. 29, n. 6 , p. 1823-1831, Nov./Dec. 2013.

SILVA, C. A. D., Prospecção em fitopatógenicos e avaliação de fontes de matéria orgânica sobre a supressividade da podridão radicular da mandioca. GaranhunsPE, 2013. 77 f. Dissertação (Mestrado em Produção Agrícola). Universidade Federal Rural de Pernambuco - Unidade Acadêmica de Garanhuns, 2013.

SILVA, H. S. A.; ANDRADE, E. C. Impacto potencial das mudanças climáticas sobre as doenças da mandioca no Brasil. In: Impactos das mudanças climáticas sobre doenças de importantes culturas no Brasil. Jaguariúna: Embrapa Meio Ambiente, p. 263-272, 2011.

SILVA, J. L.; TEIXEIRA, R. N. V. Esporulação e crescimento micelial de Fusarium solani em diferentes meios de cultura e regimes de luminosidade. Revista Agrombiente On-line,v.6,n.1,p.47-52, 2012. Disponível em: $<$ http://revista.ufrr.br/index.php/agroambiente/article/view/604>

SILVA, K. S.; REBOUÇAS, T. N. H.; BOMFIM, M. P.; SILVA, D. S.; SÃO JOSÉ, A. R.; BENETT, C. G. S. Atividade antagônica in vitro de isolados de Trichoderma spp. 
ao fungo Phytophthora citrophthora. Londrina. Semina: Ciências Agrárias, v. 29, n. 4, p. 749-754, out./dez. 2008.

SILVA, M.B.; NICOLI, A. COSTA, A.S.V.; BRASILEIRO, B.G.; JAMAL, C.M., SILVA, C. A.; PAULA JÚNIOR, T. J.; TEIXEIRA, H. Ação antimicrobiana de extratos de plantas medicinais sobre espécies fitopatogênicas de fungos do gênero Colletotrichum. Revista Brasileira de Plantas Medicinais, Botucatu, v.10, n.3, p.5760, 2008.

SOUSA, R.M.S.; SERRA, I.M.R.S.; MELO, T.A. Effect of essential oils as an alternative in the control of Colletotrichum gloesporioides in pepper.Summa Phytopathologica, v.38, n.1, p.42-47, 2012.

SOUZA, J. R., Potencialidade de fungicida e agente biológico no controle de requeima do tomateiro. 2013. 63f. Dissertação. Universidade Estadual do Sudoeste da Bahia, Vitória da Conquista. Bahia - BR. 2013.

SOUZA, K. de A.;VAZ, M. C. A.; ALVES, G. C. S.; PAZ -LIMA, M. L.. Confronto in vitro de isolados de Trichoderma spp. com fungos filamentosos. $2 p$. IV Congresso Estadual de Iniciação Científica do IF Goiano 21 a 24 de setembro de 2015.

SPIEGEL, Y.; CHET, I. Evolution of Trichoderma spp. as a biocontrol agent against soilborne fungi and plant parasitic nematodes in Israel. Integrated Pest Management Review, v. 03, p. 167-175, 1998.

STANGARLIN, J.R. et al. Controle de doenças de plantas por extratos de origem vegetal. Revisão Anual de Patologia de Plantas, v.16, p.265-304, 2008.

URBEN, A.F., et al., Curso taxonomia de Fusarium. Embrapa recursos genéticos e biotecnologia. Brasilia-DF: Embrapa informação tecnólogica, 2009.

VENTURA, J. A. Taxonomia de Fusarium e seus segregados: I- história, meios e procedimentos de cultivo. Revisão Anual de Patologia de Plantas, Passo Fundo, v. 7, p. 271-298, 1999. 
VINALE F, SIVASITHAMPARAM, K., GHISALBERTI, E. L., MARRA, R., WOO, S. L., \& LORITO, M.. Trichoderma-plant-pathogen interactions. Soil Biology and Biochemistry, v. 40, p.1-10.2008.

ZUCCHI, F. O. Trichoderma sp. em áreas agrícolas visando o controle de doenças fúngicas de raízes de plantas cultivadas. Campinas SP. JV Biotecnologia. 15p. 2010.

\section{APPENDICE - RIFERIMENTO DI FOOTNOTE}

1. WOLLENWEBER, H. W.; REINKING, O. A.. Die fusarien: ihre beschreibung, schadwirkung und bekämpfung. Berlin: P. Parey, 1935

Inviato: Maggio, 2020.

Approvato: giugno 2020. 